\title{
Erratum: Vacuum decays around spinning black holes
}

\author{
Naritaka Oshita, ${ }^{a}$ Kazushige $\mathrm{Ueda}^{b}$ and Masahide Yamaguchi ${ }^{c}$ \\ ${ }^{a}$ Perimeter Institute, \\ 31 Caroline St, Waterloo, Ontario N2L 2Y5, Canada \\ ${ }^{b}$ Graduate School of Science Hiroshima University, \\ 1-3-1 Kagami Yama, Higashi-Hiroshima, 739-8526, Japan \\ ${ }^{c}$ Department of Physics, Tokyo Institute of Technology, \\ 2-12-1 Ookayama, Meguro-ku, Tokyo 152-8551, Japan \\ E-mail: noshita@pitp.ca, m180959@hiroshima-u.ac.jp, \\ gucci@phys.titech.ac.jp
}

ERRATUM TO: JHEP01(2020)015

ARXIV EPRINT: 1909.01378

The published work relies on the diagonalized metric shown in eq. (3.7), and it was mentioned that eq. (3.7) is obtained by the transformation $d \phi \rightarrow d \psi-\tilde{g}_{\phi t} / \tilde{g}_{\phi \phi} d \lambda$. However, the diagonalized metric is not exactly obtained by the transformation but is valid approximately, which was missed in the original paper. This does not affect our results and conclusion as is explained below.

The angular velocity, $\omega \equiv-\tilde{g}_{\phi t} / \tilde{g}_{\phi \phi}$ has $r$ and $\theta$-dependence, and therefore, the correct transformation should be $d \phi \rightarrow d \psi+\omega d \lambda+\left(\partial_{r} \omega\right) \lambda d r+\left(\partial_{\theta} \omega\right) \lambda d \theta$. The last term is strongly suppressed by $\partial_{\theta} \omega \sim-4 M a^{3} \Delta / r^{7}$, which leads to negligible corrections to $\tilde{g}_{\mu \nu}$ outside the horizon. The $d r$-term in the transformation also gives a negligible correction, $C$, to the induced metric in eq. (3.11):

$$
\begin{aligned}
d s^{2} & =(-1+C) d \tau^{2}+g_{\theta \theta} d \theta^{2}+g_{\psi \psi} d \psi^{2}, \\
\text { with } \quad C & \sim 4 R^{2} \omega\left(\partial_{r} \omega\right) \dot{R} \lambda \sim-48 M^{2} a^{2} \dot{R} \lambda / R^{5},
\end{aligned}
$$

where $|C| \ll 1$ holds for $R \geq R_{\min }$. Note that although $R \sim M$ for $R \simeq R_{\min }, \dot{R} \simeq 0$ suppresses the factor $C$ there. We should also emphasize that $\lambda \lesssim R_{\max }$ is assumed since only one Euclidean period is taken into account in the calculation of the Euclidean action.

\section{Acknowledgments}

We are very grateful to Ruth Gregory for pointing out this error.

Open Access. This article is distributed under the terms of the Creative Commons Attribution License (CC-BY 4.0), which permits any use, distribution and reproduction in any medium, provided the original author(s) and source are credited. 